\title{
cAMP/PKA/CREB/GLTI signaling involved in the antidepressant-like effects of phosphodiesterase 4D inhibitor (GEBR-7b) in rats
}

This article was published in the following Dove Press journal:

Neuropsychiatric Disease and Treatment

21 January 2016

Number of times this article has been viewed

\author{
Xu Liu ${ }^{1,2, *}$ \\ Haibiao Guo 1 ,* \\ Mohammad Daud SOM \\ Sayed ${ }^{3,4, *}$ \\ Yang $\mathrm{Lu}^{3,4, *}$ \\ Ting Yang ${ }^{5, *}$ \\ Dongsheng Zhou ${ }^{6}$ \\ Zhongming Chen ${ }^{6}$ \\ Haitao Wang' \\ Chuang Wang ${ }^{3,4}$ \\ Jiangping $\mathrm{Xu}^{\mathrm{l}}$ \\ 'Guangdong Provincial Key Laboratory \\ of New Drug Screening, School of \\ Pharmaceutical Sciences, Southern Medical \\ University, Guangzhou, Guangdong, \\ ${ }^{2}$ Department of Pharmacy, General \\ Hospital of Chinese People's Armed \\ Police Forces, Beijing, ${ }^{3}$ Ningbo Key \\ Laboratory of Behavioral Neuroscience, \\ ${ }^{4}$ Zhejiang Provincial Key Laboratory of \\ Pathophysiology, School of Medicine, \\ Ningbo University, Ningbo, Zhejiang, \\ ${ }^{5}$ Department of Pediatrics, The Affiliated \\ Hospital of Ningbo University, Ningbo, \\ Zhejiang, ${ }^{6}$ Department of Geriatric \\ Psychiatry, Ningbo Kangning Hospital, \\ Ningbo, Zhejiang, People's Republic of \\ China \\ *These authors contributed equally \\ to this work
}

\begin{abstract}
Objectives: GEBR-7b, a potential phosphodiesterase 4D inhibitor, has been shown to have memory-enhancing effects in rodents. However, it is still unknown whether GEBR-7b also has the antidepressant-like effects in rats. Herein, we examined the potential of GEBR-7b to attenuate depression-like behaviors in the rat model of depression induced by chronic unpredictable stress (CUS). Next, we also investigated the alterations of cyclic adenosine monophosphate (cAMP), protein kinase A (PKA) catalytic subunit (PKAca), cAMP response element-binding (CREB), and glutamate transporter 1 (GLT1) levels produced by GEBR-7b in the rats model of depression.
\end{abstract}

Methods: Effects of GEBR-7b on CUS (35 days)-induced depression-like behaviors were examined by measuring immobility time in the forced swimming test (FST). Hippocampal cAMP levels were examined by enzyme-linked immunosorbent assay, whereas PKAca, phosphorylation of CREB (pCREB), CREB, and GLT1 in the hippocampus of rats were subjected to Western blot analysis.

Results: CUS exposure caused a depression-like behavior evidenced by the increased immobility time in FST. Depression-like behavior induced by CUS was accompanied by a significant increased GLT, decreased cAMP, PKAca, pCREB activities in hippocampus. However, repeated GEBR-7b administration significantly reversed CUS-induced depression-like behavior and changes of cAMP/PKA/CREB/GLT1 signaling. No alteration was observed in locomotor activity in open field test.

Conclusion: These findings indicate that GEBR-7b reversed the depression-like behaviors induced by CUS in rats, which is at least in part mediated by modulating cAMP, PKAca, pCREB, and GLT1 levels in the hippocampus of rats, supporting its neuroprotective potential against behavioral and biochemical dysfunctions induced by CUS

Keywords: phosphodiesterase 4D, GEBR-7b, cyclic adenosine monophosphate, protein kinase Aca, cAMP response element-binding protein, phosphorylation of CREB, glutamate transporter 1

\section{Introduction}

Depression is the most common serious brain disorder with a lifetime prevalence of up to $17 \%$. Currently available antidepressants have significant limitations, most notably low response rate and delay in onset of action. Indeed, speaking of depression, a key question has always been why therapeutic responses with antidepressants could only be achieved after at least 3-8 weeks of treatment, whereas antidepressants alter synaptic monoamine levels within hours, ${ }^{1}$ suggesting that the monoaminergic hypothesis cannot fully explain the pathophysiological mechanism of depression and the action of antidepressants. In the brain, beyond the level of monoaminergic receptor signaling, 
cyclic adenosine $3^{\prime}, 5^{\prime}$-monophosphate (cAMP) signaling has been shown to be implicated in mechanism of reduced synaptic plasticity, and neuronal survival may contribute to the pathophysiology of depression. ${ }^{2-4}$ Recently, accumulating evidence indicates that dysfunction in glutamatergic neurotransmission also contributes to the pathophysiology of depression. ${ }^{5}$ Consequently, the modulation of glutamatergic neurotransmission represents a new strategy for antidepressant development. ${ }^{6}$ To date, five excitatory amino acid transporters (EAATs), termed GLAST, GLT-1, EAAC1, EAAT4, and EAAT5, have been identified. The predominant glutamate transporter in the adult brain among the five EAATs, EAAT2, was also identified in the rodent as glutamate transporter 1 (GLT1), which was expressed predominantly in astrocytes and is responsible for $90 \%$ of total glutamate uptake to prevent neuronal excitotoxicity and hyperexcitability. ${ }^{7,8}$ In addition, given that a previous study revealed that glutamate uptake is regulated at multiple levels ${ }^{9}$ and that the expression of the transporter protein is regulated by cAMP and cAMP response element-binding protein (CREB) ${ }^{10}$ it further demonstrates that cAMP-mediated signaling may also play regulatory in the GLT1.

cAMP was originally shown to induce gene transcription through activation of cAMP-dependent protein kinase (PKA), and subsequent phosphorylation of the transcription factor CREB at serine-133. ${ }^{11}$ Growing evidence suggests that phosphodiesterase-4 (PDE4), one of eleven PDE enzyme families, is particularly important for controlling intracellular cAMP concentrations and is considered to be a prime target for therapeutic intervention for depression, ${ }^{12-14}$ and this is supported by an increasing number of animal and clinical studies. $^{12,14-18}$

Although PDE4 inhibitors have a promising antidepressant effects, no PDE4 inhibitors have yet been approved as antidepressants because of their side effects such as emesis. ${ }^{19}$ Recently, an interesting phosphodiesterase 4D inhibitor (GEBR-7b), which is a full inhibitor of PDE4D, was found. ${ }^{20}$ Compared to protypical PDE4 inhibitor rolipram, GEBR-7b was 10 times more effective in improving memory performance in healthy rodents, and reduced emesis response 100 times. $^{20}$ These results indicated that the GEBR-7b showed promise for the development of new antidepressant. In this study, we investigated whether chronic treatment with GEBR-7b could reverse the depression-like behaviors induced by chronic unpredictable stress (CUS) via restoring cAMP, PKA, and phosphorylation of CREB (pCREB). Additionally, we also examined whether GLT1 is also involved in the antidepressantlike effects of GEBR-7b in rats.

\section{Animals}

Experiments were conducted on adult, male Sprague Dawley rats (weighing 200-220 g upon arrival), which were born and reared in the animal facility of the Ningbo University Medical School, People's Republic of China. All animals were housed in a room maintained at $23^{\circ} \mathrm{C} \pm 2{ }^{\circ} \mathrm{C}$ and $60 \% \pm 5 \%$ relative humidity under a 12-hour light/12-hour dark cycle (lights on at $07 \mathrm{am}$ ) with ad libitum access to food and water when the stressors were not applied. Animals were habituated to housing conditions for 7 days prior to the beginning of experimental procedures. All stressors were applied to animals outside of their housing area in a separate procedure room. All experiments involving animals were performed according to the National Institutes of Health (NIH) Guides for the Care and Use of Laboratory Animals (NIH Publications Number 80-23, revised 1996) and were approved by the Institutional Animal Care and Use Committee of Ningbo University Medical School, People's Republic of China (Number NBU20140089).

\section{Drug}

GEBR-7b, which was purchased from Millipore (Temecula, CA, USA), was dissolved in dimethyl sulfoxide and kept at $4^{\circ} \mathrm{C}$; this stock solution was used for further dilutions in $0.5 \%$ methylcellulose. All injection solutions consisted of $0.5 \%$ methylcellulose with fixed dimethyl sulfoxide percentages $(0.01 \%$ for rats). The solution was given intraperitoneally $(0.05 \mathrm{mg} / \mathrm{kg}$, i.p.) and the injection volume was $1 \mathrm{~mL} / 100 \mathrm{~g}$.

\section{CUS paradigm and drug treatment}

Briefly, CUS consisted of exposure to a variety of unpredictable stressors (randomly), as shown in Table 1. It consisted of a variety of stressors applied randomly and at varying times of the day for 35 days. Rats were divided into three groups, ie, nonstressed rats treated with vehicle $(n=8)$, CUS rats treated with vehicle $(n=8)$, and CUS rats treated with GEBR-7b $(n=8)$. Animals in the CUS treated with vehicle group and CUS treated with GEBR-7b group were first exposed to CUS for 21 days, and then were treated with vehicle or GEBR-7b ( $0.05 \mathrm{mg} / \mathrm{kg}$, i.p.) respectively for 14 days, 30 minutes before the application of stress daily. During the 14 days of vehicle or GEBR-7b treatment, CUS rats were continuously exposed to stressors as shown in Table 1. All animals were subjected to open field test (OFT) and forced swimming test (FST) to confirm the depression-like behaviors induced by CUS. Immediately after the behavioral tests, the rats were killed by decapitation, and the hippocampi were collected for the detection of cAMP levels by enzyme-linked immunosorbent assay 
Table I Schedule of stressor used in the 35-day CUS procedure

\begin{tabular}{|c|c|}
\hline Day & Stressor \\
\hline I & Restraint stress (4 hours) \\
\hline 2 & Shaking (I hour) \\
\hline 3 & Tail pinch ( 10 minutes) \\
\hline 4 & Swim stress $\left(12^{\circ} \mathrm{C}, 10\right.$ minutes $)$ \\
\hline 5 & Constant light ( 24 hours) \\
\hline 6 & Cold swim $\left(18^{\circ} \mathrm{C}, 10\right.$ minutes $)$ \\
\hline 7 & Wet bedding ( 24 hours) \\
\hline 8 & Water deprivation ( 12 hours) \\
\hline 9 & Social isolation ( 24 hours) \\
\hline 10 & Swim stress $\left(12^{\circ} \mathrm{C}, 10\right.$ minutes $)$ \\
\hline II & Tail pinch ( 10 minutes) \\
\hline 12 & Water deprivation ( 12 hours) \\
\hline 13 & Shaking (I hour) \\
\hline 14 & Hot water stress at $42^{\circ} \mathrm{C}$ ( 5 minutes) \\
\hline 15 & Restraint stress (4 hours) \\
\hline 16 & Shaking (I hour) \\
\hline 17 & Constant light ( 24 hours) \\
\hline 18 & Social isolation ( 24 hours) \\
\hline 19 & Swim stress at $12^{\circ} \mathrm{C}(10$ minutes $)$ \\
\hline 20 & Water deprivation ( 12 hours) \\
\hline 21 & Wet bedding ( 24 hours) \\
\hline 22 & Tail pinch ( 10 minutes) \\
\hline 23 & Hot water stress at $42^{\circ} \mathrm{C}$ ( 5 minutes) \\
\hline 24 & Restraint stress ( 4 hours) \\
\hline 25 & Water deprivation ( 12 hours) \\
\hline 26 & Constant light (24 hours) \\
\hline 27 & Swim stress $\left(12^{\circ} \mathrm{C}, 10\right.$ minutes $)$ \\
\hline 28 & Open field test (5 minutes) \\
\hline 29 & Wet bedding ( 24 hours) \\
\hline 30 & Hot water stress at $42^{\circ} \mathrm{C}$ ( 5 minutes) \\
\hline 31 & Restraint stress (4 hours) \\
\hline 32 & Tail pinch (I0 minutes) \\
\hline 33 & Water deprivation ( 12 hours) \\
\hline 34 & Constant light (24 hours) \\
\hline 35 & Shaking (I hour) \\
\hline
\end{tabular}

(ELISA), and PKAca, pCREB, CREB, and GLT1 protein expression were analyzed by Western blot analysis.

\section{Behavioral testing OFT}

The OFT is a simple test used to determine general activity levels, gross locomotor activity, and exploration habits in rodent models. Assessment takes place in a square, white Plexiglas box. The rat was placed in the arena and allowed to freely move about for 5 minutes while being recorded by an overhead camera (Noldus Information Technology, Leesburg, VA, USA). After each test, the apparatus was sprayed with dilute alcohol and wiped thoroughly to eliminate the residual odor. Testing was performed in a temperature-, noise-, and light-controlled room. Total distance traveled and movement velocity were measured.
FST

Briefly, animals were placed in a cylindrical tank $(60 \mathrm{~cm}$ height, $30 \mathrm{~cm}$ diameter $)$ filled with tap water $\left(25^{\circ} \mathrm{C}\right)$ to a depth of $45 \mathrm{~cm}$ for 5 minutes. The behaviors in the FST were recorded by video cameras, one positioned on top of the swimming tank and another positioned on the side. The total time during which the rat made only small movements necessary to keep the head above water was considered the duration of immobility. To eliminate the influence of potential alarm substances, freshwater was introduced prior to each test. Trained observers blind to the drug treatments scored the video recordings.

\section{cAMP assay}

The hippocampus was dissected out bilaterally, frozen in liquid nitrogen, homogenized in ice-cold radioimmunoprecipitation assay (RIPA) lysis buffer and diluted with $0.1 \mathrm{~N}$ $\mathrm{HCl}$ to a final protein concentration of $1 \mathrm{mg} / \mathrm{mL}$, and then centrifuged at $13,000 \times g$ for 30 minutes at $4^{\circ} \mathrm{C}$. The cAMP levels from the samples were determined by ELISAassay (Assay Designs, Ann Arbor, MI, USA). The optical density was read at $405 \mathrm{~nm}$ using an ELX800 Universal Microplate Reader (Bio-TEK Instruments, Winooski, VT, USA). The cAMP concentration was expressed as $\mathrm{pmol} / \mathrm{mL}$.

\section{Western blot analysis}

Western blot analysis was performed as previously described in our study (Wang et $\mathrm{al}^{11}$ ). Briefly, hippocampal tissues were homogenized in the RIPA lysis buffer $(50 \mathrm{mM}$ Tris- $\mathrm{HCl} \mathrm{pH}$ 7.4, $150 \mathrm{mM} \mathrm{NaCl}, 1 \% \mathrm{NP}-40,0.5 \%$ sodium deoxycholate, $0.1 \%$ sodium dodecyl sulfate; Upstate Biotechnology, Temecula, CA, USA) containing protease and phosphatase inhibitors (Pierce Biotechnology, Rockford, IL, USA) and then centrifuged at $15,000 \times g$ for 30 minutes. Samples $(80 \mu \mathrm{g}$ protein each) were separated using sodium dodecyl sulfate-polyacrylamide gel electrophoresis and subsequently transferred to polyvinylidene difluoride membranes $(0.22 \mu \mathrm{m}$; Millipore). The samples were then incubated overnight with rabbit anti-pCREB (Ser133) (1:1,000; Millipore), anti-CREB (1:1,000; Millipore), GLT1 (1:1,000; Millipore), anti-PKAca antibody (1:1,000; Abcam, Burlingame, CA, USA), and anti- $\beta$-actin antibodies (1:1,000; Cell Signaling, Danvers, MA, USA) at $4^{\circ} \mathrm{C}$. Afterward, the membranes were incubated with Alexa Fluor 700conjugated goat anti-rabbit antibody $(1: 10,000$; Invitrogen, OR, USA) for 60 minutes. Detection and quantification of specific bands were performed using a fluorescence scanner (Odyssey Infrared Imaging System, LI-COR Biotechnology, Lincoln, NE, USA). For band stripping, the membranes were 
incubated with a stripping buffer (Chemicon, Temecula, CA, USA) for 15 minutes.

\section{Statistical analyses}

Data are expressed as the means \pm standard error of means and statistically analyzed by one-way analysis of variance (ANOVA) followed by the Newmane-Keuls multiple comparison test using the commercially available GraphPad Prism 5.0 software (GraphPad Software, San Diego, CA, USA). A $P$-value $<0.05$ was considered to indicate statistical significance.

\section{Results}

\section{Effects of GEBR-7b on CUS-induced depression-like behaviors in rats}

The time course of stress exposure, drug treatment, and behavioral tests is shown in Figure 1A. For behavioral tests, one-way ANOVA indicated significant differences among three treatments in their effect on open field behaviors: exploratory activity $[F(2,21)=11.53, P=0.0004$; Figure $1 \mathrm{~B}]$ and movement velocity $[F(2,21)=91.92, P<0.0001$; Figure $1 C]$.
In addition, post hoc test revealed that CUS rats treated with vehicle exhibited a significant decrease in exploration $(P<0.01)$ and movement velocity $(P<0.01)$ compared with the nonstressed rats treated with vehicle; however, GEBR-7b treatment had no significant effect on open field measures when CUS rats treated with GEBR-7b were compared with CUS rats treated with vehicle. In the FST, one-way ANOVA showed significant effects of treatment with GEBR-7b on immobility time $[F(2,21)=8.891, P=0.0016$; Figure 1D]. A post hoc test demonstrated that GEBR-7b treatment significantly decreased immobility time $(P<0.01)$ in CUS rats treated with GEBR-7b (Figure 1D) compared with CUS rats treated with vehicle. Reversal of CUS-induced "behavioral despair" by 14-day treatment with GEBR-7b confirmed the antidepressant-like effects of GEBR-7b.

\section{Effects of GEBR-7b on the levels of cAMP and expression of PKAca, PCREB, CREB, and GLTI in the hippocampus of rats}

As shown in Figure 2, CUS significantly decreased the levels of cAMP $[F(2,21)=19.16, P<0.0001$; Figure 2] and the
A
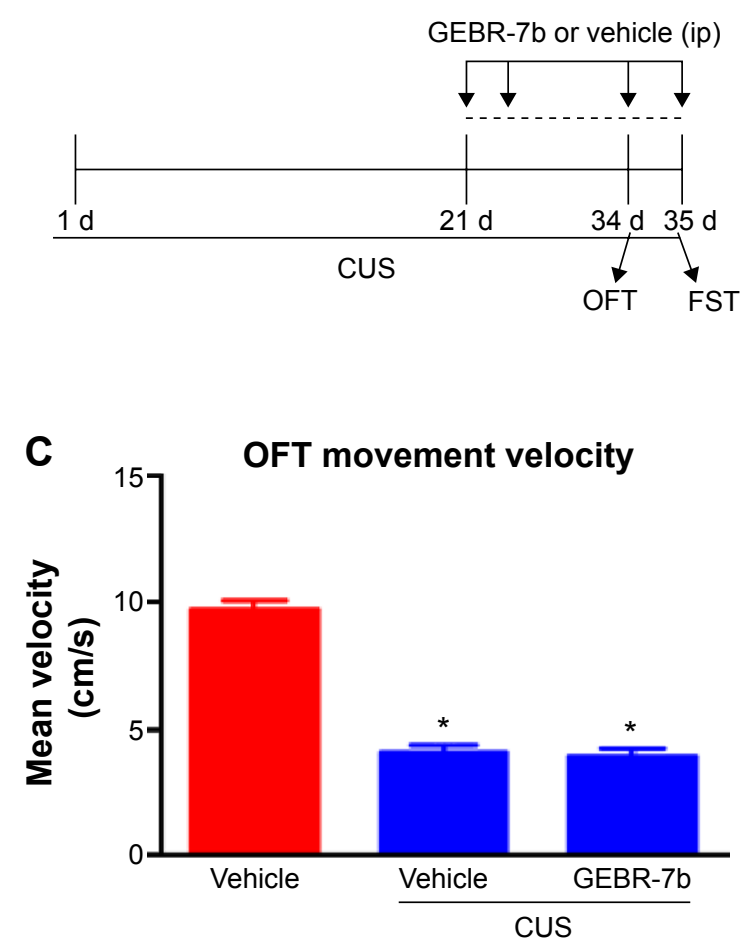

B OFT travelled distance

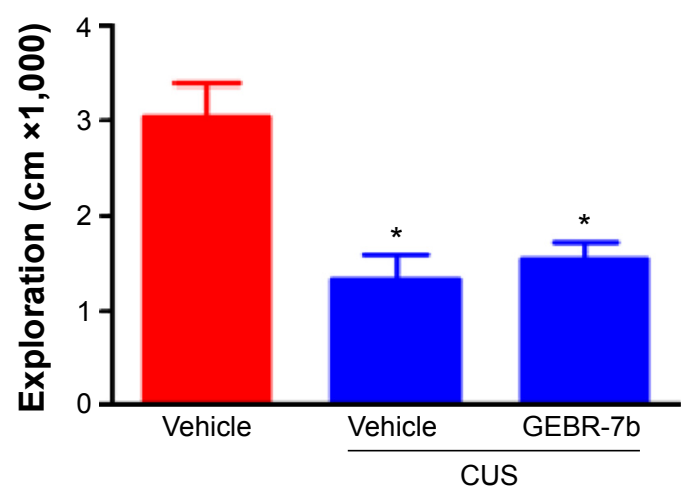

D

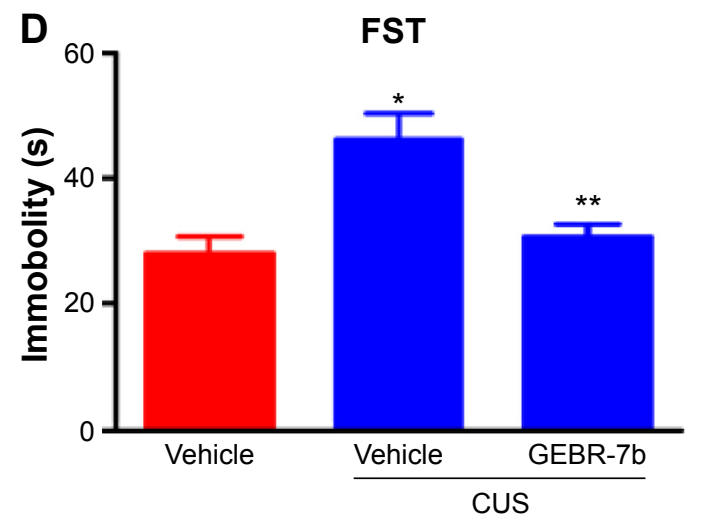

Figure I Effect of chronic GEBR-7b treatment on CUS-induced depression-like behaviors in rats.

Notes: (A) Schematic representation of the experimental procedure for CUS and treatments in rats. CUS rats were exposed to one stressor per day for $2 \mathrm{I}$ days, and then received 14 days of GEBR-7b or vehicle injections during which CUS continued. (B) OFT: exploratory activities (total distance traveled) were evaluated in a 5-minute test session. (C) OFT: movement velocity was evaluated in a 5-minute test session. (D) FST: time spent for immobility was scored for a 5-minute-test session. Results are expressed as mean $\pm S E M$ ( $n=8$ per group). $* P<0.0$ I, compared to nonstressed rats treated with vehicle; $* * P<0.05$.

Abbreviations: CUS, chronic unpredictable stress; OFT, open field test; FST, forced swimming test; SEM, standard error of the mean; ip, intraperitoneal injection. 


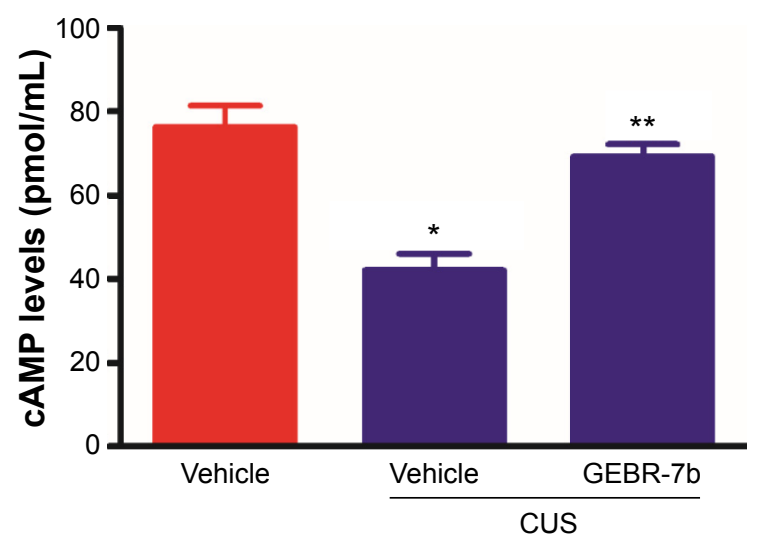

Figure 2 Chronic GEBR-7b treatment reverses chronic unpredictable stress (CUS)induced reduction of CAMP levels in the hippocampus of rats.

Notes: Data shown are means \pm SEM of eight animals/group, and these were analyzed using one-way ANOVA followed by Newman-Keuls tests. $* P<0.01$ vs nonstressed rats treated with vehicle; $* * P<0.01$ versus stressed rats treated with vehicle.

Abbreviations: CUS, chronic unpredictable stress; cAMP, cyclic adenosine monophosphate; SEM, standard error of the mean; ANOVA, analysis of variance. expression of PKAca $[F(2,21)=8.453, P=0.0020$; Figure 3B $]$, pCREB $[F(2,21)=34.74, P<0.0001$; Figure $3 \mathrm{C}]$, and GLT1 $[F(2,21)=13.05, P=0.0002$; Figure $3 \mathrm{E}]$ in the hippocampus compared with nonstressed rats treated with vehicle group. In addition, GEBR-7b $(0.05 \mathrm{mg} / \mathrm{kg}$, ip) significantly reversed the CUS-induced changes in cAMP $(P<0.01)$, PKAca $(P<0.01)$, pCREB $(P<0.01)$, and GLT1 $(P<0.01)$ in the hippocampus of rats. However, none of the treatments affected the expression of CREB $[F(2,21)=0.4413, P=0.6490$; Figure $3 \mathrm{D}$ ] in the hippocampus of rats.

\section{Discussion}

In this study, we demonstrated that 2-week treatment with GEBR-7b significantly reversed CUS-induced depressionlike behaviors and also restored the CUS-induced downregulation of cAMP, PKAca, pCREB, and GLT1 in hippocampus

A
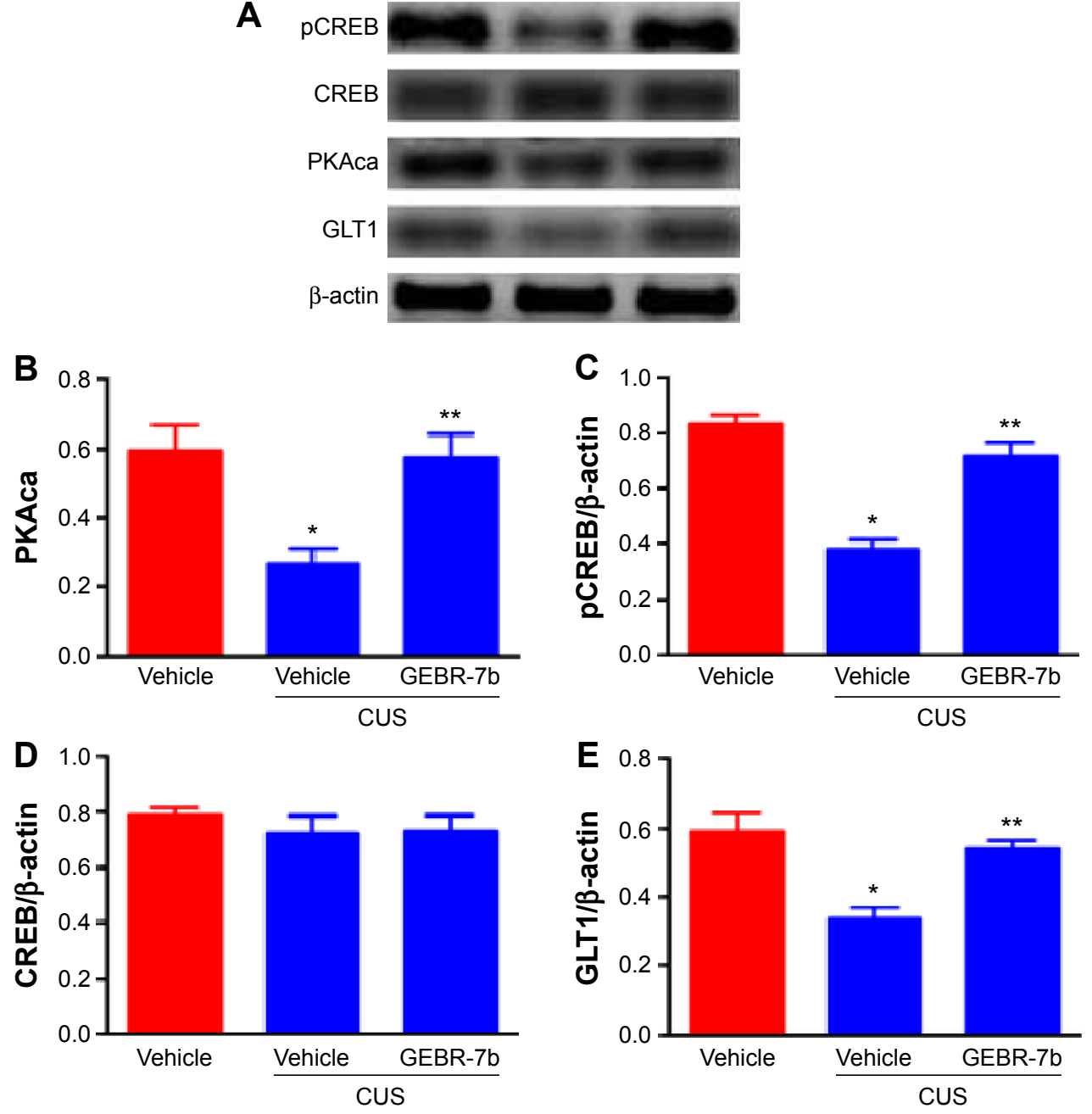

Figure 3 Effect of chronic GEBR-7b treatment on expression of PKAca, PCREB, CREB, and GLTI in the hippocampus of rats.

Notes: (A) Representative immunoblots of PKAca, PCREB, CREB, and GLTI detected by Western blotting with tissues from the hippocampus; the rest of the panels are the quantification of the immunoblotting bands of PKAca (B), PCREB (C), CREB (D), and GLTI (E). Data shown are mean \pm SEM of eight animals/group and were analyzed using one-way ANOVA followed by Newman-Keuls tests. $* P<0.01$ vs nonstressed rats treated with vehicle; $* * P<0.01$ vs stressed rats treated with vehicle.

Abbreviations: PKAca, protein kinase A catalytic subunit; PCREB, phosphorylation of cAMP response element-binding; CREB, cAMP response element-binding; GLTI, glutamate transporter I; cAMP, cyclic adenosine monophosphate; CUS, chronic unpredictable stress; SEM, standard error of the mean. 
of rats. Given that the selection of hippocampus was based on the evidence that it plays an important role in the regulation of mood disorders, ${ }^{21,22}$ our current data may suggest a potential relationship between antidepressant-like effects of GEBR-7b and the hippocampus.

The CUS procedure is one of the well-validated animal models of depression and mimics the stressful events common in human society. ${ }^{15}$ Based on our preliminary data, the 35-day CUS duration was chosen in our present study. Additionally, the depression-like behavior was examined by the FST, which is widely employed as a behavioral tool to determine depression-like behaviors in animals after exposure to $\mathrm{CUS}^{23}$ This task is also quite sensitive to all major classes of antidepressant drugs including tricyclics, serotonin-specific reuptake inhibitors, monoamine oxidase inhibitors, and atypicals. ${ }^{24-26}$ Further, an evaluation of locomotor and exploratory activities by OFT was also conducted to exclude mismatch factors to the FST. In this parameter, it was observed that the reduction in the immobility time in the FST elicited by GEBR-7b was not accompanied by alterations on the locomotor activity. Our findings provided further confirmation that PDE4D inhibitor may play an important role in antidepressant actions.

The inhibition of PDE4 enzyme is a way to augment the second messenger cAMP transduction pathway, and PDE4 has been studied as a potential target for treating depression for more than 20 years. ${ }^{27}$ Four different subtypes of PDE4 have been cloned and are termed PDE4A, PDE4B, PDE4C, and PDE4D, with multiple splice variants for each gene. ${ }^{28}$ PDE4 inhibitors have been widely investigated for their antidepressant effects in both animals and humans ${ }^{14,29}$ and, within this context, PDE4D has been proposed as the PDE subtype responsible for the antidepressant effects of rolipram. ${ }^{30}$ However, despite its potential clinical relevance, the therapeutic use of rolipram is limited because of the acute emesis occurring after its administration. ${ }^{31}$ Recently, a growing body of data has shown that the novel selective PDE4D inhibitor GEBR-7b is able to improve the late-phase consolidation processes of spatial and object recognition memory and increase the hippocampal levels of cAMP in vivo. ${ }^{20}$ Consistent with the previous results obtained, the results of this study showed that the cAMP levels in the hippocampus were significantly increased by GEBR-7b. Furthermore, our current study also provided a novel report that the GEBR-7b significantly reversed the depression-like behaviors induced by CUS in rats.

Earlier studies revealed that glutamatergic neurotransmission is involved in the pathogenesis of stress and depression-like behaviors. $^{32,33}$ The previous studies demonstrated that the intracerebroventricular infusion of the astrocytic glutamate reuptake inhibitor dihydrokainic acid produces depressionlike behaviors in rats, ${ }^{34}$ indicating that glutamate uptake may play a critical role in the pathophysiology of depression. Given that cAMP mediates the increase in glutamate uptake in both slices and cultured astrocytes, and cellular activity appears to be necessary for cAMP to be effective in both slices and cultured astrocytes, ${ }^{35}$ it is important to further clarify whether the GLT1 is also involved in the antidepressant effects of GEBR-7b. To the best of our knowledge, this is the first report showing that GLT1 involved in cAMP/PKA/CREB signaling and antidepressant-like effects of GEBR-7b.

Interestingly, in the present work, the downregulation of GLT1 induced by CUS could be reversed by chronic GEBR-7b administration, suggesting that GLT1 may be involved in the antidepressant actions of GEBR-7b. Consistent with the previous work reported by Zink et al, ${ }^{36}$ our results shown that the GLT1 levels were reduced in the hippocampus of rats by CUS. However, in contrast with a previous study which demonstrated that chronic stress increased GLT1 immunoreactivity, ${ }^{37}$ GLT1 mRNA levels and GLT1 protein expression increased in the hippocampus of rats following 21 days of CUS. ${ }^{38,39}$ Although the mechanisms responsible for these CUS-induced upregulation of GLT1 are unclear, our current work employed the CUS (35-day) regimen of varied stressors for a longer duration than the 21-day regimen, which was used in previous works, ${ }^{37-39}$ and this may explain the inconsistency. Therefore, the increase of GLT1 levels may occur as a compensatory response to stress-induced elevations in glutamate. In support of this hypothesis, the application of glutamate to primary glial cultures dose-dependently increases GLT1 mRNA. ${ }^{40}$ However, a series of previous studies conducted by Banasr et $\mathrm{al}^{41-43}$ and Czeh et $\mathrm{al}^{44}$ have demonstrated that CUS for 35 days in rats significantly suppressed cell proliferation in the cerebral cortex, ${ }^{43}$ induced the reduction in glial cell density in the prefrontal cortex of adult rats, ${ }^{42}$ and decreased the glial fibrillary acidic protein (GFAP, a key cytoskeletal protein of astrocytes) mRNA expression in the prefrontal cortex ${ }^{41}$ and the number of GFAP-positive cells in the hippocampus. ${ }^{44}$ With an increased stress intensity and a prolonged stress duration time, elevated GLT1 is not sufficient to resist the rising glutamate in the synaptic cleft. Therefore, excessive synaptic cleft glutamate inhibits or even damages the function of astrocytes, ${ }^{41-43}$ which leads to the decreased expression of GLT1 and further strengthens glutamate excitotoxicity. However, the precise mechanisms of different CUS duration 


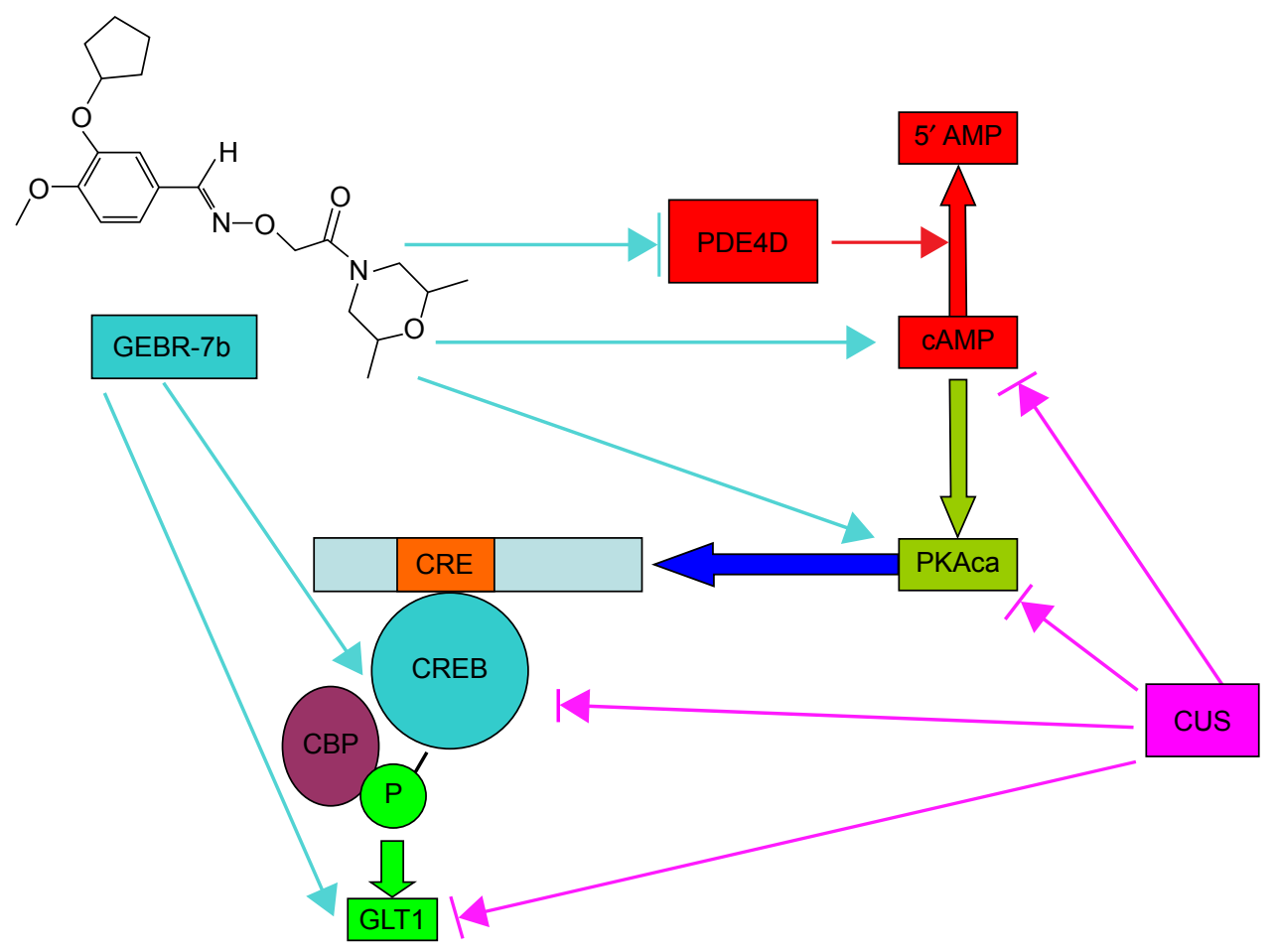

Figure 4 Diagram of signaling pathway indicating the possible mechanism by which GEBR-7b reversed CUS-induced depression-like behaviors in rats. Notes: GEBR-7b inhibited the PDE4D activity, and further increased the levels cAMP, PKA, and pCREB, resulting in the upregulation of GLTI in hippocampus of rats. $\rightarrow$ indicates activate/induce. $\rightarrow$ indicates inhibit/antagonism.

Abbreviations: CUS, chronic unpredictable stress; PDE4D, phosphodiesterase 4D; cAMP, cyclic adenosine monophosphate; PKA, protein kinase A; pCREB, phosphorylation of cAMP response element-binding; GLTI, glutamate transporter I; PKAca, protein kinase A catalytic subunit; CBP, CREB binding protein.

that changes the GLT1 levels need to be further clarified. In addition, Hughes et $\mathrm{l}^{45}$ suggested that GFAP might play a pivotal role in PKA-induced GLT1 trafficking at the astrocytic membrane in the hippocampus, indicating that cAMP/PKA/pCREB signaling may be involved in the upregulation of GLT1 produced by GEBR-7b.

Taken together, the results of this study suggest that GEBR-7b can reverse the downregulation of cAMP/ PKA/pCREB/GLT1 signaling under the chronic stress condition and improve depression-like behaviors of rats (The possible mechanism of GEBR-7b reversed CUS-induced depression-like behaviors in rats is shown in Figure 4). Based on the aforementioned findings, our finding further concluded that GEBR-7b may be an innovative candidate for an antidepressant. However, further studies are warranted to examine the possible mechanism(s) of GLT1's involvement in the antidepressant effects of GEBR-7b and are also need to clarify whether GLT1 is a key downstream mediator of cAMP/PKA/CREB signaling in the antidepressant-like activity of GEBR-7b.

\section{Acknowledgments}

This work was supported by National Natural Science Foundation of China (Number 81201050; Number 81271209, and Number 81371224); Natural Science Foundation of Zhejiang province (Number LQ12H09001); Natural Science Foundation of Ningbo (Number 2012A610249); The Open Research Fund of State Key Laboratory of Bioelectronics, Southeast University (To Junfang Zhang) and Zhejiang "Climbing" Program (PD2013104). Student Research and Innovation Program (SRIP) of Ningbo University. This project was also sponsored by KC Wong Magna funded at Ningbo University.

\section{Disclosure}

The authors report no conflicts of interest in this work.

\section{References}

1. Kreiss DS, Lucki I. Effects of acute and repeated administration of antidepressant drugs on extracellular levels of 5-hydroxytryptamine measured in vivo. J Pharmacol Exp Ther. 1995;274(2):866-876.

2. Wang ZZ, Zhang Y, Liu YQ, et al. RNA interference-mediated phosphodiesterase 4D splice variants knock-down in the prefrontal cortex produces antidepressant-like and cognition-enhancing effects. $\mathrm{Br}$ J Pharmacol. 2013;168(4):1001-1014.

3. Li YF, Cheng YF, Huang Y, et al. Phosphodiesterase-4D knock-out and RNA interference-mediated knock-down enhance memory and increase hippocampal neurogenesis via increased cAMP signaling. $J$ Neurosci. 2011;31(1):172-183.

4. Manji HK, Duman RS. Impairments of neuroplasticity and cellular resilience in severe mood disorders: implications for the development of novel therapeutics. Psychopharmacol Bull. 2001;35(2):5-49. 
5. Mitchell ND, Baker GB. An update on the role of glutamate in the pathophysiology of depression. Acta Psychiatr Scand. 2010;122(3): 192-210.

6. Catena-Dell'Osso M, Fagiolini A, Rotella F, Baroni S, Marazziti D. Glutamate system as target for development of novel antidepressants. CNS Spectr. 2013;18(4):188-198.

7. Sheldon AL, Robinson MB. The role of glutamate transporters in neurodegenerative diseases and potential opportunities for intervention. Neurochem Int. 2007;51(6-7):333-355.

8. Maragakis NJ, Dykes-Hoberg M, Rothstein JD. Altered expression of the glutamate transporter EAAT2b in neurological disease. Ann Neurol. 2004;55(4):469-477.

9. Gegelashvili G, Dehnes Y, Danbolt NC, Schousboe A. The high-affinity glutamate transporters GLT1, GLAST, and EAAT4 are regulated via different signalling mechanisms. Neurochem Int. 2000;37(2-3): 163-170.

10. Karki P, Webb A, Smith K, et al. cAMP response element-binding protein $(\mathrm{CREB})$ and nuclear factor $\mathrm{\kappa B}$ mediate the tamoxifen-induced up-regulation of glutamate transporter 1 (GLT-1) in rat astrocytes. J Biol Chem. 2013;288(40):28975-28986.

11. Wang C, Yang XM, Zhuo YY, et al. The phosphodiesterase-4 inhibitor rolipram reverses $\mathrm{A} \beta$-induced cognitive impairment and neuroinflammatory and apoptotic responses in rats. Int J Neuropsychopharmacol. 2012;15(6):749-766.

12. Jindal A, Mahesh R, Bhatt S. Type 4 phosphodiesterase enzyme inhibitor, rolipram rescues behavioral deficits in olfactory bulbectomy models of depression: involvement of hypothalamic-pituitary-adrenal axis, cAMP signaling aspects and antioxidant defense system. Pharmacol Biochem Behav. 2015;132(132):20-32.

13. Guo J, Lin P, Zhao X, et al. Etazolate abrogates the lipopolysaccharide (LPS)-induced downregulation of the $\mathrm{cAMP} / \mathrm{pCREB} / \mathrm{BDNF}$ signaling, neuroinflammatory response and depression-like behavior in mice. Neuroscience. 2014;28(163):1-14.

14. Zhang MZ, Zhou ZZ, Yuan X, et al. Chlorbipram: a novel PDE4 inhibitor with improved safety as a potential antidepressant and cognitive enhancer. Eur J Pharmacol. 2013;721(1-3):56-63.

15. Mahar I, Bambico FR, Mechawar N, Nobrega JN. Stress, serotonin, and hippocampal neurogenesis in relation to depression and antidepressant effects. Neurosci Biobehav Rev. 2014;38:173-192.

16. Halene TB, Siegel SJ. PDE inhibitors in psychiatry - future options for dementia, depression and schizophrenia? Drug Discov Today. 2007; 12(19-20):870-878.

17. Saccomano NA, Vinick FJ, Koe BK, et al. Calcium-independent phosphodiesterase inhibitors as putative antidepressants:[3-(bicycloalkyloxy)4-methoxyphenyl]-2-imidazolidinones. J Med Chem. 1991;34(1): 291-298.

18. Fleischhacker WW, Hinterhuber H, Bauer H, et al. A multicenter doubleblind study of three different doses of the new cAMP-phosphodiesterase inhibitor rolipram in patients with major depressive disorder. Neuropsychobiology. 1992;26(1-2):59-64.

19. Burgin AB, Magnusson OT, Singh J, et al. Design of phosphodiesterase 4D (PDE4D) allosteric modulators for enhancing cognition with improved safety. Nat Biotechnol. 2010;28(1):63-70.

20. Bruno O, Fedele E, Prickaerts J, et al. GEBR-7b, a novel PDE4D selective inhibitor that improves memory in rodents at non-emetic doses. Br J Pharmacol. 2011;164(8):2054-2063.

21. Xu Y, Sheng H, Tang Z, Lu J, Ni X. Inflammation and increased IDO in hippocampus contribute to depression-like behavior induced by estrogen deficiency. Behav Brain Res. 2015;288:71-78.

22. Malykhin NV, Coupland NJ. Hippocampal neuroplasticity in major depressive disorder. Neuroscience. Epub April 28, 2015.

23. Porsolt RD, Le Pichon M, Jalfre M. Depression: a new animal model sensitive to antidepressant treatments. Nature. 1977;266(5604):730-732.

24. Doboszewska U, Szewczyk B, Sowa-Kucma M, et al. Antidepressant activity of fluoxetine in the zinc deficiency model in rats involves the NMDA receptor complex. Behav Brain Res. 2015;287:323-330.
25. Villégier AS, Gallager B, Heston J, Belluzzi JD, Leslie FM. Age influences the effects of nicotine and monoamineoxidase inhibition on mood-related behaviors in rats. Psychopharmacology. 2010;208(4): 749-766.

26. Rodrigues AL, da Silva GL, Mateussi AS, et al. Involvement of monoaminergic system in the antidepressant-like effect of the hydroalcoholic extract of Siphocampylus verticillatus. Life Sci. 2002;70(12): 1347-1358.

27. O'Donnell JM, Zhang HT. Antidepressant effects of inhibitors of cAMP phosphodiesterase (PDE4). Trends Pharmacol Sci. 2004;25(3): 158-163.

28. Houslay MD, Sullivan M, Bolger GB. The multienzyme PDE4 cyclic adenosine monophosphate-specific phosphodiesterase family: intracellular targeting, regulation, and selective inhibition by compounds exerting anti-inflammatory and antidepressant actions. Adv Pharmacol. 1998; 44:225-342.

29. Terburg D, Syal S, Rosenberger LA, et al. Acute effects of Sceletium tortuosum (Zembrin), a dual 5-HT reuptake and PDE4 inhibitor, in the human amygdala and its connection to the hypothalamus. Neuropsychopharmacology. 2013;38(13):2708-2716.

30. Zhang HT, Huang Y, Jin SL, et al. Antidepressant-like profile and reduced sensitivity to rolipram in mice deficient in the PDE4D phosphodiesterase enzyme. Neuropsychopharmacology. 2002;27(4): 587-595.

31. Hebenstreit GF, Fellerer K, Fichte K, et al. Rolipram in major depressive disorder: results of a double-blind comparative study with imipramine. Pharmacopsychiatry. 1989;22(4):156-160.

32. Merega E, Pittaluga A, Nicoletti F, et al. The effects of antidepressant treatment in prenatally stressed rats support the glutamatergic hypothesis of stress-related disorders. J Neurosci. 2014;34(6): 2015-2024.

33. Duman RS. Pathophysiology of depression and innovative treatments: remodeling glutamatergic synaptic connections. Dialogues Clin Neurosci. 2014;16(1):11-27.

34. Bechtholt-Gompf AJ, Walther HV, Adams MA, Carlezon WA Jr, Ongur D, Cohen BM. Blockade of astrocytic glutamate uptake in rats induces signs of anhedonia and impaired spatial memory. Neuropsychopharmacology. 2010;35(10):2049-2059.

35. Pita-Almenar JD, Collado MS, Colbert CM, Eskin A. Different mechanisms exist for the plasticity of glutamate reuptake during early long-term potentiation (LTP) and late LTP. J Neurosci. 2006;26(41): 10461-10471

36. Zink M, Vollmayr B, Gebicke-Haerter PJ, Henn FA. Reduced expression of glutamate transporters vGluT1, EAAT2 and EAAT4 in learned helpless rats, an animal model of depression. Neuropharmacology. 2010;58(2):465-473.

37. Raudensky J, Yamamoto BK. Effects of chronic unpredictable stress and methamphetamine on hippocampal glutamate function. Brain Res. 2007;1135(1):129-135.

38. Reagan LP, Rosell DR, Wood GE, et al. Chronic restraint stress upregulates GLT-1 mRNA and protein expression in the rat hippocampus: reversal by tianeptine. Proc Natl Acad Sci U S A. 2004;101(7): 2179-2184

39. Lowy MT, Gault L, Yamamoto BK. Adrenalectomy attenuates stressinduced elevations in extracellular glutamate concentrations in the hippocampus. J Neurochem. 1993;61(5):1957-1960.

40. Thorlin T, Roginski RS, Choudhury K, et al. Regulation of the glial glutamate transporter GLT-1 by glutamate and delta-opioid receptor stimulation. FEBS Lett. 1998;425(3):453-459.

41. Banasr M, Chowdhury GM, Terwilliger R, et al. Glial pathology in an animal model of depression: reversal of stress-induced cellular, metabolic and behavioral deficits by the glutamate-modulating drug riluzole. Mol Psychiatry. 2010;15(5):501-511.

42. Banasr M, Duman RS. Glial loss in the prefrontal cortex is sufficient to induce depression-like behaviors. Biol Psychiatry. 2008; 64(10):863-870. 
43. Banasr M, Valentine GW, Li XY, Gourley SL, Taylor JR, Duman RS. Chronic unpredictable stress decreases cell proliferation in the cerebral cortex of the adult rat. Biol Psychiatry. 2007;62(5):496-504.

44. Czeh B, Simon M, Schmelting B, Hiemke C, Fuchs E. Astroglial plasticity in the hippocampus is affected by chronic psychosocial stress and concomitant fluoxetine treatment. Neuropsychopharmacology. 2006; 31(8):1616-1626.
45. Hughes EG, Maguire JL, McMinn MT, Scholz RE, Sutherland ML. Loss of glial fibrillary acidic protein results in decreased glutamate transport and inhibition of PKA-induced EAAT2 cell surface trafficking. Brain Res Mol Brain Res. 2004;124(2):114-123.

\section{Publish your work in this journal}

Neuropsychiatric Disease and Treatment is an international, peerreviewed journal of clinical therapeutics and pharmacology focusing on concise rapid reporting of clinical or pre-clinical studies on a range of neuropsychiatric and neurological disorders. This journal is indexed on PubMed Central, the 'PsycINFO' database and CAS, and is the official journal of The International Neuropsychiatric Association (INA). The manuscript management system is completely online and includes a very quick and fair peer-review system, which is all easy to use. Visit http://www.dovepress.com/testimonials.php to read real quotes from published authors.

Submit your manuscript here: http://www.dovepress.com/neuropsychiatric-disease-and-treatment-journal 